\title{
Thread-assisted mucosal defect closure using the locking-clip technique after gastric endoscopic submucosal dissection
}

Methods of closing mucosal defects after gastric endoscopic submucosal dissection (ESD) have been reported [1-3]. However, closing large mucosal defects in the stomach is considered difficult compared with other parts of the intestinal tract because the submucosal layer is thick [4]. We designed the threadassisted closure method using a lockingclip technique (LCT) for closing mucosal defects.

Thread-assisted closure using LCT uses clips and a thread (commercially available dental floss). After setting the clip, the thread is fixed on the proximal side of the clip's tooth ( $\triangleright$ Fig.1). LCT involves the clip and thread becoming strongly fixed when fully opened. First, a threaded clip is attached to the marginal defect edge followed by the mucosal defect margin on the contralateral side. Next, LCT is performed by pulling the thread and bringing the mucosal defect margins close to each other ( $\triangleright$ Video 1 ). The mucosal defect margins are fixed, and the defect base is completely closed by repeating the technique.

We closed a mucosal defect of approximately $50 \mathrm{~mm}$ in size after gastric ESD using thread-assisted closure via LCT. First, the endoscope was pulled out from the stomach, a clip fixed with the thread was attached, and the scope was reinserted. The first clip was attached to the marginal part of the defect on the anal side. Subsequently the second clip was attached to the mucosal defect margin. Finally, LCT was used to completely fix the margins ( $>$ Fig. 2 , > Video 1 ). Repeated LCT helped perform thread-assisted closure multiple times to completely close the defect. The patient was discharged uneventfully.

Thread-assisted closure and line-assisted complete closure are effective methods for closing mucosal defects. By using $\mathrm{LCT}$, it was possible to perform threadassisted closure several times with a sin-


- Fig. 1 Locking-clip technique device. a After setting the clip from the applicator, the thread is fixed to the proximal side of the clip's tooth. $\mathbf{b}$ The fixed thread stiffens and sticks to the clip.

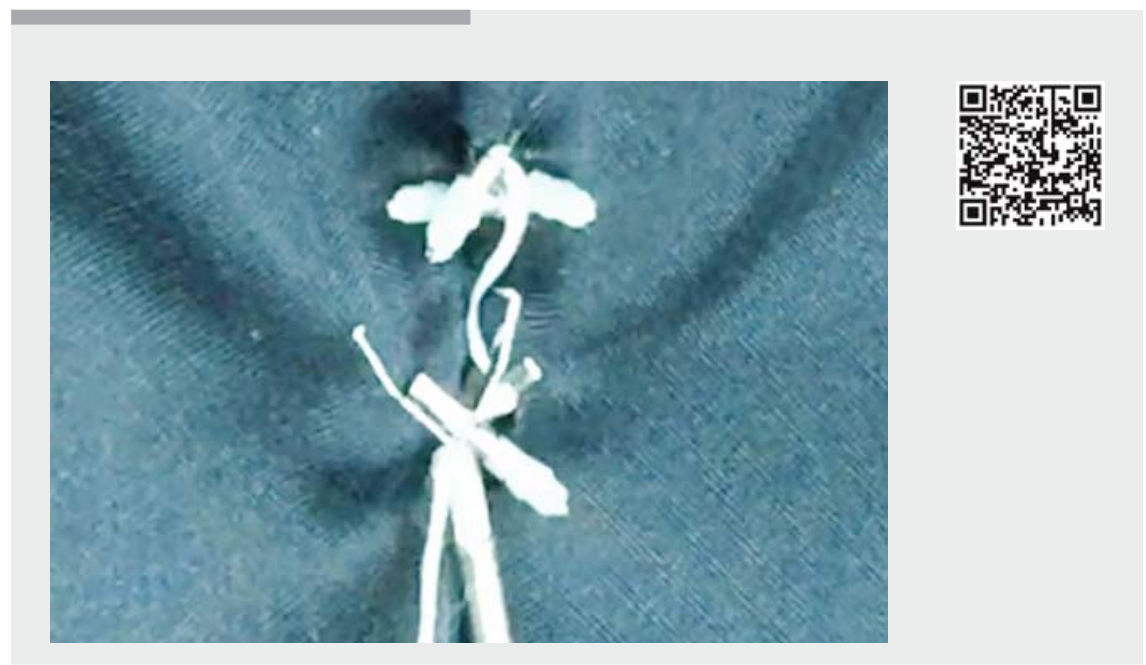

$\checkmark$ Video 1 Thread-assisted mucosal defect closure using the locking-clip technique after endoscopic submucosal dissection.

gle thread and bring rigid mucosal defect Endoscopy_UCTN_Code_TTT_1AO_2AG 


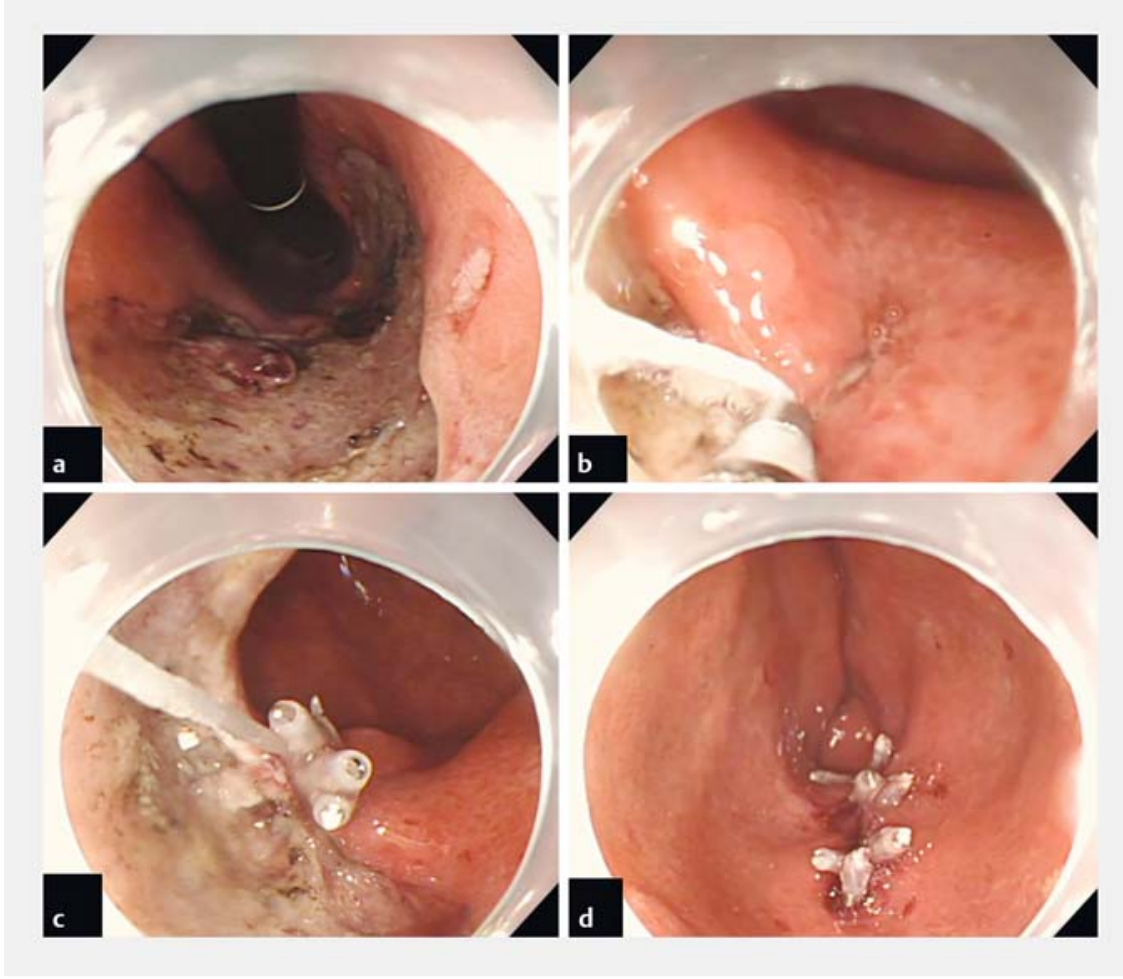

- Fig. 2 Locking-clip technique. a Mucosal defect after gastric endoscopic submucosal dissection. b A threaded clip is attached to the marginal defect edge. $\mathbf{c}$ Thread-assisted mucosal closure using the locking-clip technique. $\mathbf{d}$ Completely closed mucosal defect after gastric endoscopic submucosal dissection.

\section{Competing interests}

None

\section{The authors}

Tatsuma Nomura ${ }^{1,2}$, Akira Kamei ${ }^{2}$, Shinya Sugimoto ${ }^{2}$, Tetsuro Harada ${ }^{1}$, Jun Oyamda ${ }^{2}$

1 Department of Gastroenterology, Kinan Hospital, Minamimuro, Mie, Japan

2 Department of Gastroenterology, Ise Red Cross Hospital, Ise, Mie, Japan

\section{References}

[1] Yamasaki Y, Takeuchi Y, Kato M et al. Lineassisted complete closure of large gastric mucosal defects by use of multiple clip-andline technique. VideoGIE 2016; 1: 49-50

[2] Mönkemüller K, Peter S, Toshniwal J et al. Multipurpose use of the 'bear claw' (overthe-scope-clip system) to treat endoluminal gastrointestinal disorders. Dig Endosc 2014; 26: $350-357$

[3] Nomura T, Kamei A, Sugimoto $S$ et al. New modified hook device for endoloop closure of the mucosal defect after gastric endoscopic submucosal dissection. Endoscopy 2018; 50: E222 -E223

[4] Maekawa S, Nomura R, Murase T et al. Complete closure of artificial gastric ulcer after endoscopic submucosal dissection by combined use of a single over the scope clip and through the scope clips. Surg Endosc 2015; 29: $500-504$

\section{Bibliography}

DOI https://doi.org/10.1055/a-0725-7754

Published online: 27.9.2018

Endoscopy 2018; 50: E342-E343

(c) Georg Thieme Verlag KG

Stuttgart · New York

ISSN 0013-726X

\section{ENDOSCOPY E-VIDEOS}

https://eref.thieme.de/e-videos

\section{Tatsuma Nomura, MD}

Department of Gastroenterology, Kinan Hospital, 4750 Atawa, Mihama-cho, Minamimuro-gun, Mie 519-5293, Japan Fax: $+815-9792-3357$ m06076tn@icloud.com 